\title{
HmsB enhances biofilm formation in Yersinia pestis
}

\author{
Nan Fang ${ }^{\dagger}$, Shi $\mathrm{Qu}^{+}$, Huiying Yang, Haihong Fang, Lei Liu, Yiquan Zhang, Li Wang, Yanping Han, \\ Dongsheng Zhou * and Ruifu Yang *
}

State Key Laboratory of Pathogen and Biosecurity, Beijing Institute of Microbiology and Epidemiology, Beijing, China

\section{Edited by:}

Yi-Cheng Sun, Chinese Academy of

Medical Sciences and Peking Union

Medical College, China

Reviewed by:

Jinlei Zhao, University of

Pennsylvania, USA

Houhui Song, Zhejiang Agriculture

and Forestry University, China

\section{*Correspondence:}

Dongsheng Zhou and Ruifu Yang,

State Key Laboratory of Pathogen

and Biosecurity, Beijing Institute of

Microbiology and Epidemiology,

No. 20, Dongdajie, Fengtai, Beijing

100071, China

e-mail:dongshengzhou1977@

gmail.com

ruifuyang@gmail.com

tThese authors have contributed

equally to this work.
The hmsHFRS operon is responsible for biosynthesis and translocation of biofilm matrix exopolysaccharide. Yersinia pestis expresses the two sole diguanylate cyclases HmsT and $\mathrm{HmsD}$ and the sole phosphodiesterase HmsP, which are specific for biosynthesis and degradation, respectively, of $3^{\prime}, 5^{\prime}$-cyclic diguanosine monophosphate (c-di-GMP), a second messenger promoting exopolysaccharide production. In this work, the phenotypic assays indicates that $Y$. pestis sRNA HmsB enhances the production of c-di-GMP, exopolysaccharide, and biofilm. Further gene regulation experiments disclose that $\mathrm{HmsB}$ stimulates the expression of $h m s B, h m s C D E, h m s T$, and $h m s H F R S$ but represses that of hmsP. HmsB most likely acts as a major activator of biofilm formation in Y. pestis. This is the first report of regulation of Yersinia biofilm formation by a sRNA. Data presented here will promote us to gain a deeper understanding of the complex regulatory circuits controlling Yersinia biofilm formation.

Keywords: Yersinia pestis, HmsB, c-di-GMP, biofilm

\section{INTRODUCTION}

Yersinia pestis is the causative agent of plague, one of the most dangerous infectious diseases. Flea-borne transmission of Y. pestis occurs among mammals including humans, which distinguishes this pathogen from its genetically close progenitor Y. pseudotuberculosis that is a mild food-borne enteric pathogen (Zhou and Yang, 2011). Y. pestis biofilms, a population of bacterial colonies embedded in a self-produced exopolysaccharide matrix (Darby, 2008; Hinnebusch and Erickson, 2008; Zhou and Yang, 2011), can attach to and physically block flea's proventriculus. The inability to take in a blood meal when the proventriculus is blocked makes fleas feel hungry and bite repeatedly and thereby promoting $Y$. pestis to be spread into new individuals of mammalian reservoirs (Darby, 2008; Hinnebusch and Erickson, 2008; Zhou and Yang, 2011).

Yersinia pestis biofilms can also block feeding of model nematode Caenorhabditis elegans, because attached biofilms are primarily found on the larva head to blanket the mouth (Darby et al., 2002; Fang et al., 2013). By contrast, most strains of Y. pseudotuberculosis have the biofilm-negative phenotype, although a few of them (being similar to $Y$. pestis) can form robust biofilms at gas-liquid-solid interfaces or on nematodes (Erickson et al., 2006; Fang et al., 2013).

hmsHFRS, hmsCDE, hmsT, and $h m s P$ encode the major factors involved in biofilm formation of $Y$. pestis. HmsHFRS are responsible for biosynthesis and translocation of exopolysaccharide through cell envelope (Bobrov et al., 2008; Hinnebusch and Erickson, 2008), HmsT and HmsD are the two sole diguanylate cyclases, which are responsible for biosynthesis of $3^{\prime}, 5^{\prime}$-cyclic diguanosine monophosphate (c-di-GMP), a second messenger promoting exopolysaccharide production (Bobrov et al., 2011; Sun et al., 2011). HmsP is the sole phosphodiesterase responsible for c-di-GMP degradation (Kirillina et al., 2004; Bobrov et al., 2005).

cDNA cloning approach and deep sequencing technology have been used for global identification of small RNA (sRNA) candidates in Y. pestis (Qu et al., 2012; Beauregard et al., 2013; Yan et al., 2013; Schiano et al., 2014) and Y. pseudotuberculosis (Koo et al., 2011). However, only three specific sRNA, namely Ysr141 (Schiano et al., 2014), GcvB (McArthur et al., 2006), and RyhB (Deng et al., 2014), have been characterized for their contribution to gene regulation in $Y$. pestis.

Our previous RNA-seq study of Y. pestis (Yan et al., 2013) identified a temperature-dependent sRNA named sRNA035 located nearby $h m s C D E$ (Figure 1), which promoted us to test whether sRNA035 was involved in regulating the production of $\mathrm{c}$-di-GMP and biofilm formation. This sRNA was designated HmsB herein. This follow-up study disclosed that HmsB positively regulated $h m s C D E, h m s T, h m s H F R S$ and its own gene but negatively regulated $h m s P$, and thus acted as a major activator of c-di-GMP, exopolysaccharide and biofilm production in $Y$. pestis.

\section{EXPERIMENTAL PROCEDURE BACTERIAL STRAINS}

The wild-type Yersinia pestis Microtus strain 201 (WT) is avirulent to humans but highly virulent to mice (Zhou et al., 2004). The partial coding region of each indicated gene was replaced by the kanamycin resistance cassette by using the one-step inactivation method based on the lambda phage recombination system (Datsenko and Wanner, 2000), to generate the corresponding 


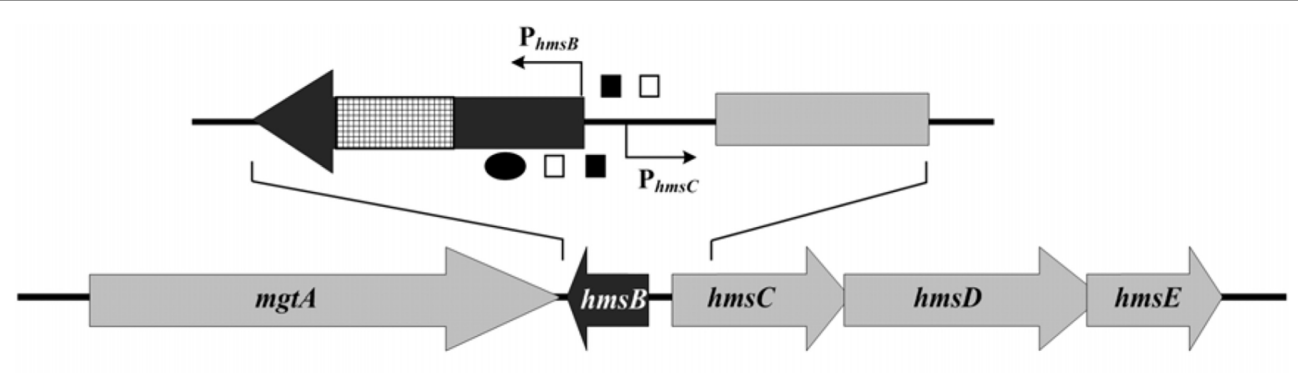

Region of deletion for construction of $\triangle \mathrm{hms} B$

-10 region $\square-35$ region

ResAB box-like sequence

FIGURE 1 | Genetic organization of $\boldsymbol{h m s} \boldsymbol{B}$ and $\boldsymbol{h m s} \boldsymbol{C D E}$. Boxed arrows represented length and direction of indicated ORFs. Broken arrows indicated transcription starts (i.e., transcribed promoters). Please refer to our companion submission for regulatory action of Rcs $A B$ on $h m s B$ and $h m s C D E$.

Table 1 | Y. pestis strains involved in gene deletion and complementation.

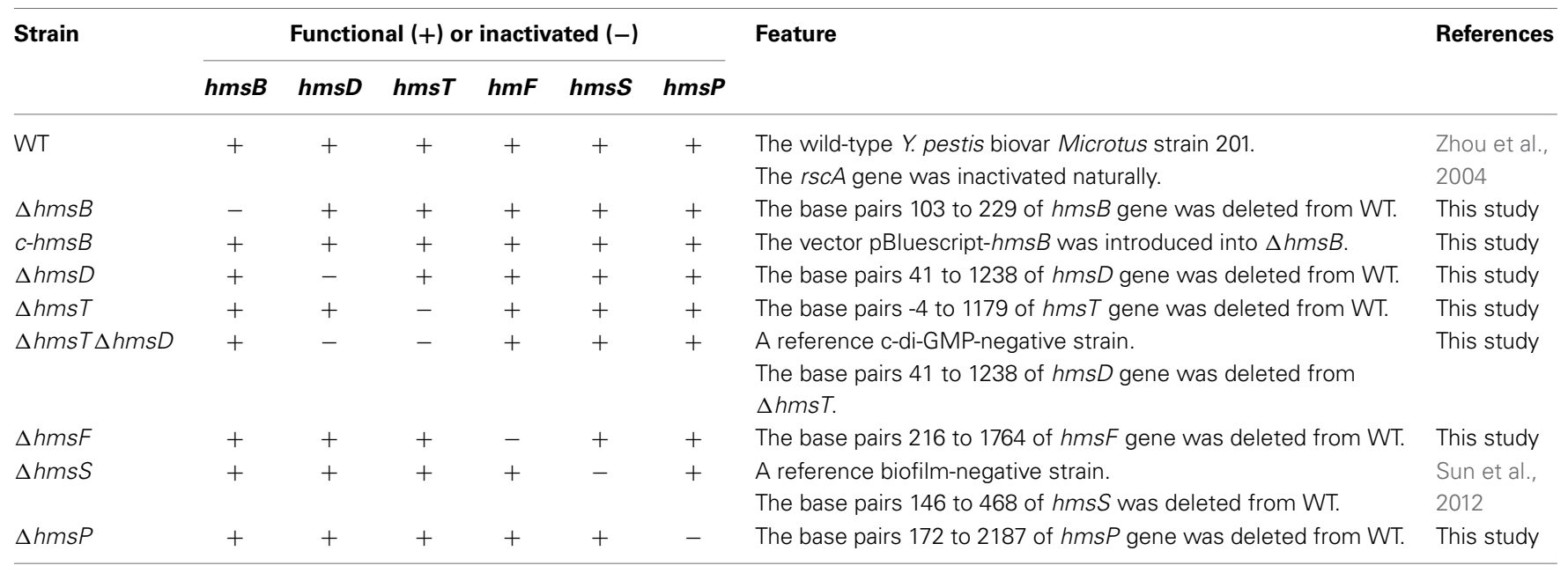

mutant of $Y$. pestis (Table 1). For in trans complementation, a PCR-generated DNA fragment containing the coding region of each indicated gene together with its promoter-proximal region and transcriptional terminator-proximal region was cloned into the cloning vector pBluescript II KS(+) (Agilent Technologies). The resulting recombinant vector was transformed into indicated $Y$. pestis strain lack of the corresponding functional gene, generating the corresponding complemented mutant (Table 1). All the primers designed in this study were listed in Table S1.

\section{BACTERIAL GROWTH AND RNA ISOLATION}

Overnight cell cultures in the Luria-Bertani (LB) broth with an optical density $\left(\mathrm{OD}_{620}\right)$ of about 1.0 were diluted 1:50 into $18 \mathrm{ml}$ of fresh $\mathrm{LB}$ broth for further cultivation at $26^{\circ} \mathrm{C}$ with shaking at $230 \mathrm{rpm}$ to reach the middle stationary phases $\left(\mathrm{an} \mathrm{OD}_{620}\right.$ of $0.8-1.2$ ), followed by cell harvest for further gene regulation or phenotypic assays. Immediately before bacterial harvest for RNA isolation, double-volume of RNAprotect reagent (Qiagen) was added to one-volume of cell culture, and total RNA was extracted using TRIzol Reagent (Invitrogen). RNA quality was monitored by agarose gel electrophoresis, and RNA quantity was determined by spectrophotometry.

\section{5'-RACE AND 3'-RACE}

Following generation of cDNA sample from total RNA through reverse transcription (RT), 5' - or $3^{\prime}$-rapid amplification of cDNA ends was done using SMARTer RACE cDNA Amplification Kit. After agarose gel electrophoresis, the $5^{\prime}$-RACE or $3^{\prime}$-RACE fragment was recovered and purified with TaKaRa MiniBEST Agarose Gel DNA Extraction Kit, and sequenced with ABI-3700 automated DNA sequencer.

\section{PRIMER EXTENSION ASSAY}

As described in our previous studies (Sun et al., 2012; Zhang et al., 2013a,b), a 5'-32 P-labeled oligonucleotide primer complementary to a portion of the RNA transcript of each indicated gene was employed to synthesize cDNAs from total RNA templates using Promega Primer Extension System. If different Y. pestis strains were involved in a single experiment, equal amounts of total RNA 
were used as starting materials. Sequence ladders were prepared with the same $5^{\prime}-{ }^{32} \mathrm{P}$-labeled primers using AccuPower \& Top DNA Sequencing Kit (Bioneer). Radioactive species were detected by autoradiography. The $5^{\prime}$-terminus of RNA transcript (i.e., transcription start) of each target gene was mapped according to size of primer extension product, while the relative mRNA levels were determined with intensities of primer extension product.

\section{LacZ FUSION AND $\beta$ GALACTOSIDASE ASSAY}

A promoter-proximal DNA region of each indicated gene was cloned into the low-copy-number transcriptional fusion vector pRW50 (Lodge et al., 1992) that harbors a promoterless lacZ reporter gene. $Y$. pestis strains transformed with the recombinant plasmid or the empty pRW50 (negative control) were grown to measure $\beta$-galactosidase activity in cellular extract using $\beta$ Galactosidase Enzyme Assay System (Promega) (Sun et al., 2012; Zhang et al., 2013a,b).

\section{ANTIBODY PREPARATION AND WESTERN BLOT}

The $6 \times$ His-tagged peptide fragments of HmsT (a.a.285-390), HmsD (a.a.221-425), HmsF (a.a.193-482), and HmsP (a.a.441671) were over-expressed, respectively, in BL21 (DE3) cells using pET28a vectors. Each recombinant protein was purified under denaturing conditions with Ni-NTA Agarose Column, and further prepared as soluble protein sample after renaturation for further immunization of New Zealand rabbits. The specific polyclonal IgG antibody was separated from rabbit serum by ammonium sulfate precipitation. For Western blot, cleared whole-cell lysate was prepared from harvested bacterial cells through sonication, followed by determination of protein concentrations with Bio-Rad protein assay kit. If different $Y$. pestis strains were involved in a single experiment, equal amounts of protein sample were separated on SDS-PAGE, immunoblotted to polyvinylidene fluoride membranes (Immobilon P; Millipore), and incubated with primary antibody and then goat anti-rabbit IRDye ${ }^{\circledR} 800 \mathrm{CW}$ second antibody. Signals were detected with Odyssey Sa Infrared Imaging System.

\section{BIOFILM AND c-di-GMP ASSAYS}

As described in our previous study (Fang et al., 2013), three different methods were used to detect $Y$. pestis biofilms. First, in vitro biofilm masses, attached to well walls when bacteria were grown in polystyrene microtiter plates, were stained with crystal violet. Second, percentages of fourth-stage larvae and adults (L4/adult) of C. elegans after incubation of nematode eggs on $Y$. pestis lawns, negatively reflecting bacterial ability to produce biofilms, were determined. Third, rugose colony morphology of bacteria grown on LB agar plates, positively reflecting bacterial ability to synthesize exopolysaccharide, was observed. In addition, intracellular c-di-GMP levels were determined by a chromatography-coupled tandem mass spectrometry (HPLC-MS/MS) method as described in our previous study (Sun et al., 2012).

\section{EXPERIMENTAL REPLICATES AND STATISTICAL METHODS}

For LacZ fusion, crystal violet staining of biofilms, and determination of L4/adult nematodes or c-di-GMP, experiments were performed with at least three independent bacterial cultures/lawns, and values were expressed as mean \pm standard deviation. Paired Student's $t$-test was performed to determine statistically significant differences; $P<0.01$ was considered to indicate statistical significance. For primer extension, Western blot, and colony morphology observation, representative data from at least two independent bacterial cultures were shown.

\section{RESULTS}

The $5^{\prime}$ and $3^{\prime}$ termini of HmsB were determined by $5^{\prime}$-RACE and $3^{\prime}$-RACE, respectively. The 262-bp $h m s B$ gene, situated from nucleotide position 4,72,430-4,72,691 on Y. pestis CO92 genome, was located within the intergenic region of mgtA (YPO0451) and $h m s C$, and the two adjacent genes $h m s B$ and $h m s C$ were transcribed with opposite direction (Figure 1).

Determination of the growth curves of WT, $\Delta h m s B$ and $c$ $h m s B$ showed that the $h m s B$ deletion had no affect on bacterial growth in vitro (data not shown). Crystal violet could steadily stain in vitro biofilm masses produced by WT or $c-h m s B$; by contrast, $\Delta h m s B$ stained a great deal less crystal violet (Figure 2A), and as expected, almost no crystal violet straining could be detected for the reference biofilm-negative strain $\Delta h m s S$. After incubation of nematode eggs on bacterial lawns of WT or $c$ $h m s B$, only a small portion (below 20\%) of larvae grew and developed to L4/adult nematodes due to abundant attachment of $Y$. pestis biofilms on nematode heads; by contrast, bacterial lawns of $\Delta h m s B$ and $\Delta h m s S$ gave the percentage values of about 65 and $100 \%$, respectively (Figure 2B). These indicated that the $h m s B$ deletion compromised biofilm formation both in vitro and on nematodes. When grow on agar plates, WT and $c$-hms $B$ gave similar rugose colony morphology due to abundant biosynthesis of exopolysaccharide, $\Delta h m s S$ produced very smooth colonies, while $\Delta h m s B$ lied between $\Delta h m s S$ and WT/c-hmsB (Figure 2C). Intracellular c-di-GMP concentrations were determined in WT, $\Delta h m s B$, and $c-h m s B$ by a HPL-MC/MS method. Compared to WT or $c-h m s B$, a significantly decreased production of c-di-GMP was observed for $\Delta h m s B$ (Figure 2D). As expected, almost no cdi-GMP could be detected for the reference c-di-GMP-negative strain $\Delta h m s T \Delta h m s D$. In addition, there were similar observations of bacterial growth curve, c-di-GMP concentration, and crystal violet staining of biofilms in WT, $\Delta h m s B$ and $c-h m s B$ (data not shown), when bacteria were grown in Brain Heart Infusion (BHI) broth or in chemically defined TMH medium (Straley and Bowmer, 1986). Taken together, the above results indicated that HmsB enhances c-di-GMP and exopolysaccharide production, which could account for HmsB-dependent lesion of biofilm formation.

$h m s T, h m s H F R S, h m s C D E, h m s P$ and its own gene were subjected to the following gene regulation assays for characterization of HmsB-dependent expression of these target genes. Levels of gene expression and protein biosynthesis were determined in WT and $\Delta h m s B$ but not the complemented mutant strain $c-h m s B$. This design was based on the following two observations: $c-h m s B$ and WT gave very similar c-di-GMP and biofilm phenotypes (see above); and no change in expression of $h m s C$ (upstream of $h m s B$ ) or $m g t A$ (downstream) was detected in $c$-hmsB relative to WT by using quantitative RT-PCR and primer extension (data not shown). 


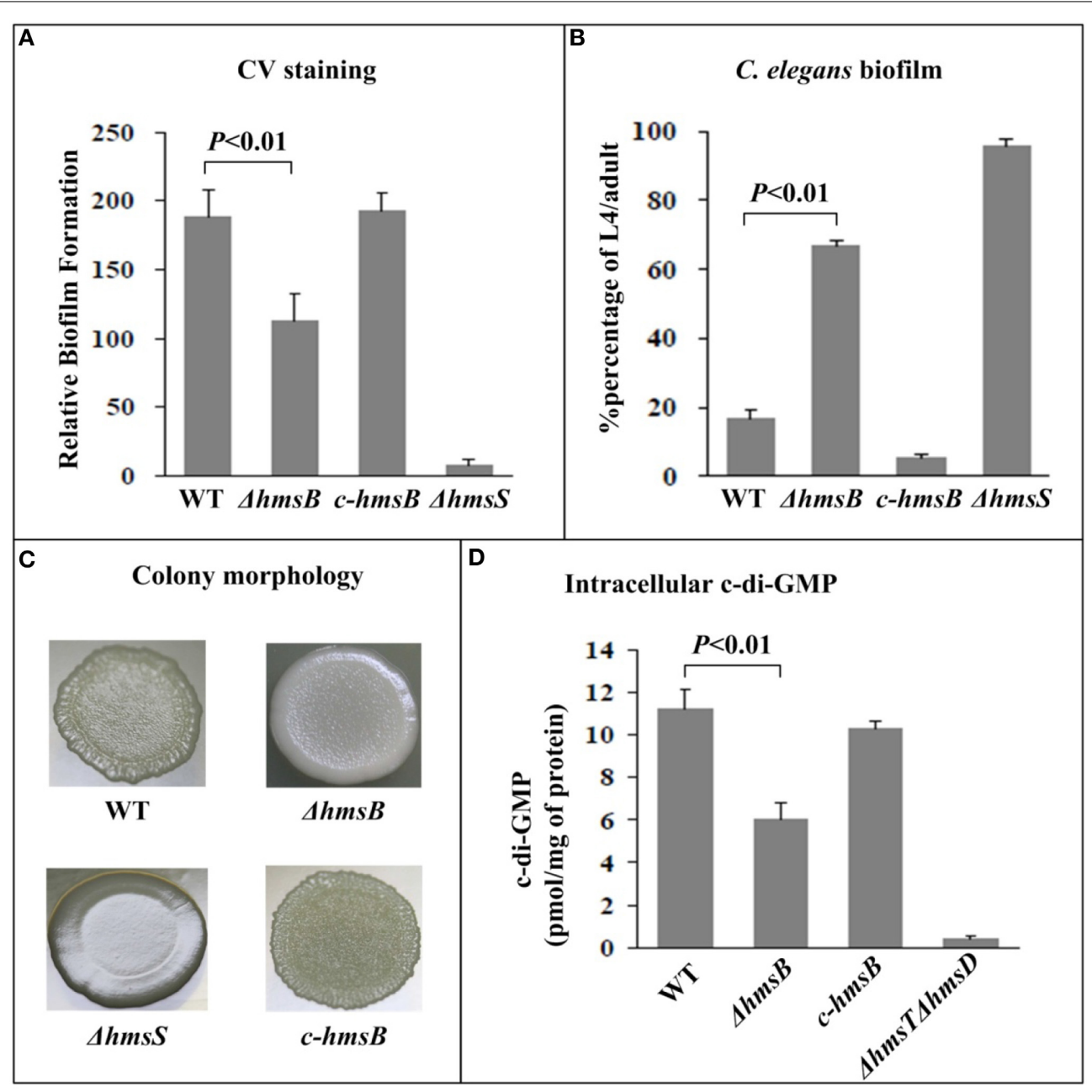

FIGURE 2 | Involvement of HmsB in biofilm formation and c-di-GMP biosynthesis. (A) Crystal violet staining. Y. pestis was grown in 24-well polystyrene dishes, and the bacterial biomass (in vitro biofilms) attached to well walls were stained with crystal violet to determine $O_{570}$ values. The planktonic cells were subjective to determine $\mathrm{OD}_{620}$ values. The relative capacity of biofilm formation of each strain tested was shown with values of $500 \times \mathrm{OD}_{570} / \mathrm{OD}_{620}$. (B) C. elegans biofilms. After incubation of nematode eggs on lawns of indicated $Y$. pestis strains, the developmental stages of nematodes on each lawn were scored to calculate percentage of L4/adult. (C) Bacterial colony morphology. Aliquots of bacterial glycerol stocks were spotted on LB plate, followed by incubation for one week. (D) Intracellular c-di-GMP concentration. The intracellular c-di-GMP concentrations were determined by a HPLC-MS/MS method, and the determining values were expressed as $\mathrm{pmol} / \mathrm{mg}$ of bacterial protein.
The relative mRNA level of each of $h m s B$ (Figure 3A), $h m s C$ (Figure 4A), $h m s T$ (Figure 5A), and $h m s H$ (Figure 6A) was measured in WT or $\triangle h m s B$ by primer extension assay, and the results showed that the mRNA level of each of the above four genes decreased considerably in $\Delta h m s B$ relative to WT. Notably, this assay detected a single transcription start site (nucleotide A) located at nucleotide position 472430 on CO92 genome, which confirmed the above $5^{\prime}$-RACE result. The promoter-proximal region of each of $h m s B$ (Figure 3B), $h m s C$ (Figure 4B), $h m s T$ (Figure 5B), and $h m s H$ (Figure 6B) was cloned into the transcriptional lacZ fusion reporter vector pRW50, and the corresponding recombinant vector was introduced into WT or $\Delta h m s B$ to determine the target promoter activity; it was shown that the promoter activity of each of the above four genes was significantly reduced in $\triangle h m s B$ relative to WT. Further Western blot assay confirmed that biosynthesis of each of HmsD (Figure 4C), HmsT
(Figure 5C), and HmsF (Figure 6C) decreased in $\triangle h m s B$ relative to WT. Notably, observations from transcriptional lac $Z$ fusion experiments denoted that HmsB-dependent expression of $h m s B$, $h m s C D E, h m s T$, and $h m s H F R S$ most likely involved mechanisms of gene transcriptional regulation.

By contrast, primer extension (Figure 7A) and Western blot (Figure 7B) assays indicated negative regulation of $h m s P$ by $\mathrm{HmsB}$ at mRNA and protein levels, respectively. Further transcriptional lac $Z$ fusion experiments (Figure 7C) indicated that $\mathrm{HmsB}$ had no regulatory effect on $h m s P$ promoter activity.

\section{DISCUSSION}

Data presented here showed that $Y$. pestis sRNA HmsB enhanced the production of c-di-GMP, exopolysaccharide, and biofilm. In addition, HmsB stimulated expression of $h m s B$, hmsCDE, hmsT, and hmsHFRS, all of which encoded biofilm-enhancing factors, 


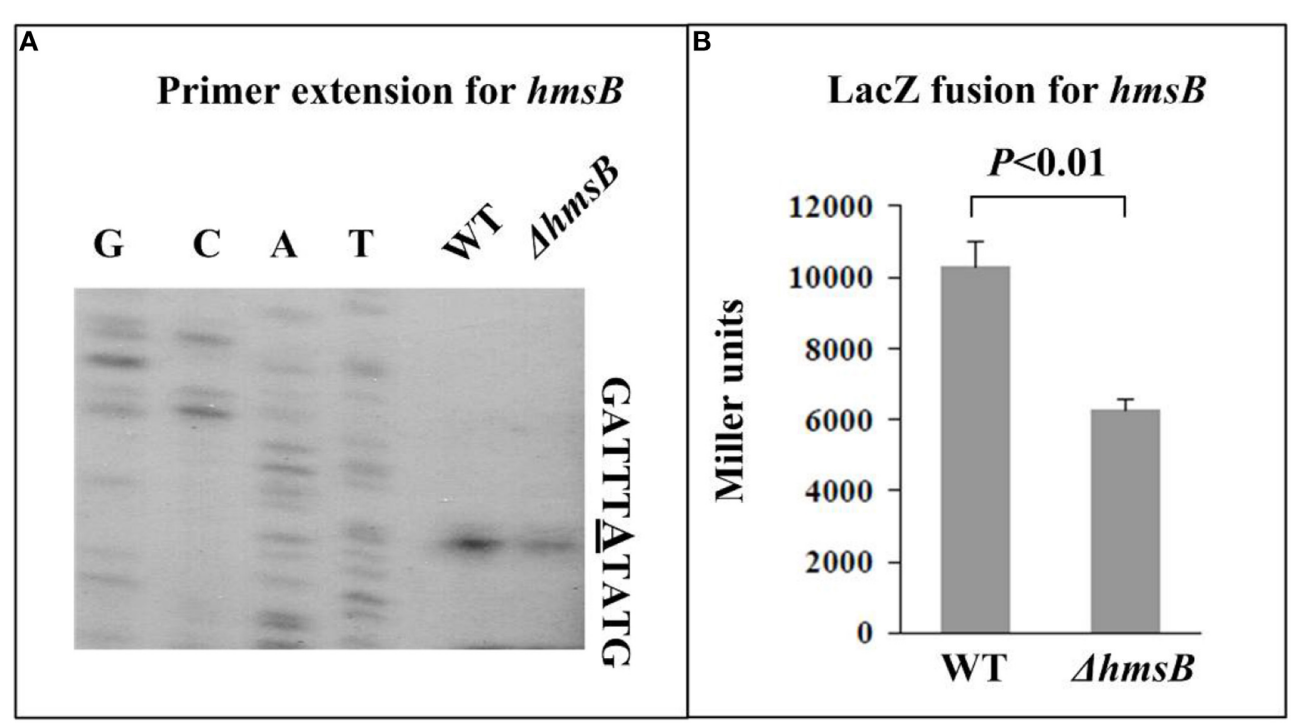

FIGURE 3 | HmsB-dependent expression of hmsB. (A) Primer extension. The mRNA levels of $h m s B$ in WT or $\Delta h m s B$ were determined by primer extension. The Sanger sequence ladders (lanes $\mathrm{G}, \mathrm{C}, \mathrm{A}$, and T) and the primer extension products of $h m s B$ were analyzed with an $8 \mathrm{M}$ urea- $6 \%$ acrylamide sequencing gel. The transcription start site of $h m s B$ was indicated by underlined nucleotide A. (B) LacZ fusion. The PhmsB:lacZ transcriptional fusion vector was transformed into WT or $\Delta h m s B$, and then the $h m s B$ promoter activities (miller units of $\beta$-galactosidase activity) were determined in bacterial cellular extracts.

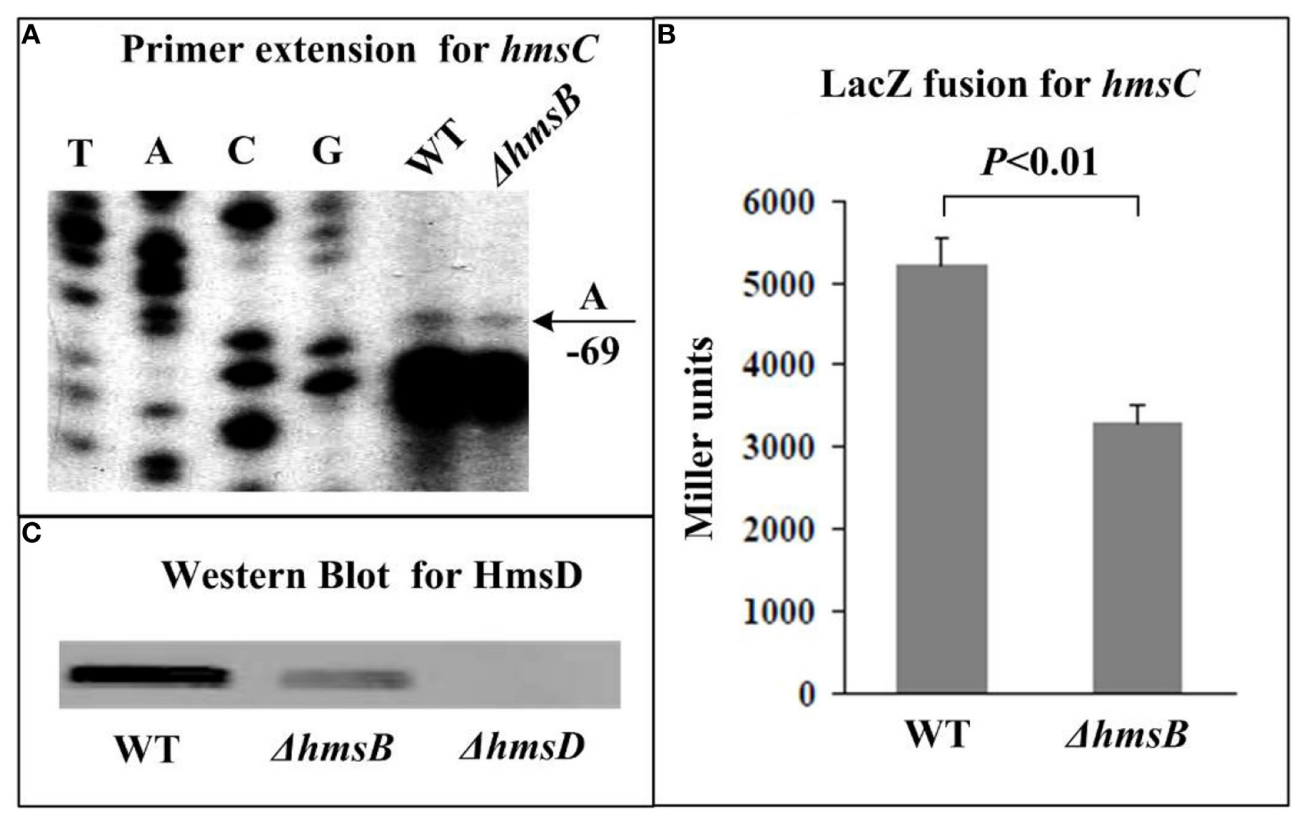

FIGURE 4 | HmsB-dependent expression of $\boldsymbol{h} \boldsymbol{m} \boldsymbol{s} \boldsymbol{C D E}$. Primer extension (A) and LacZ fusion (B) experiments were done for $h m s C$ as described in

Figure 3. The $5^{\prime}$ terminus of RNA transcript (i.e., transcription start) of $h m s C$ was indicated by arrow with nucleotide $A$, and the minus numbers under arrow indicated nucleotide position upstream of hmsC. For Western Blot (C), whole-cell protein extract from WT or $\Delta h m s B$ or $\Delta h m s D$ (negative control) was loaded for SDS-PAGE and incubated with anti-HmsD antibodies. Noted that the first gene of $h m s C E D$ was subjected to gene expression assays (A,B), while diguanylate cyclase HmsD was chosen for protein biosynthesis analysis (C). while repressed that of $h m s P$ encoding a biofilm-inhibiting factor. HmsB appeared to act as a major activator of biofilm formation in Y. pestis. To the best of our knowledge, this is the first report of a sRNA regulating Yersinia biofilm formation.
HmsB had regulatory effect on promoter activity of $h m s B$, $h m s C D E, h m s T$, and $h m s H F R S$ but not that of $h m s P$. Commonly, sRNAs inhibit the translation of their mRNA targets by base paring with the neighborhoods of ribosomal binding sites (RBSs) to 


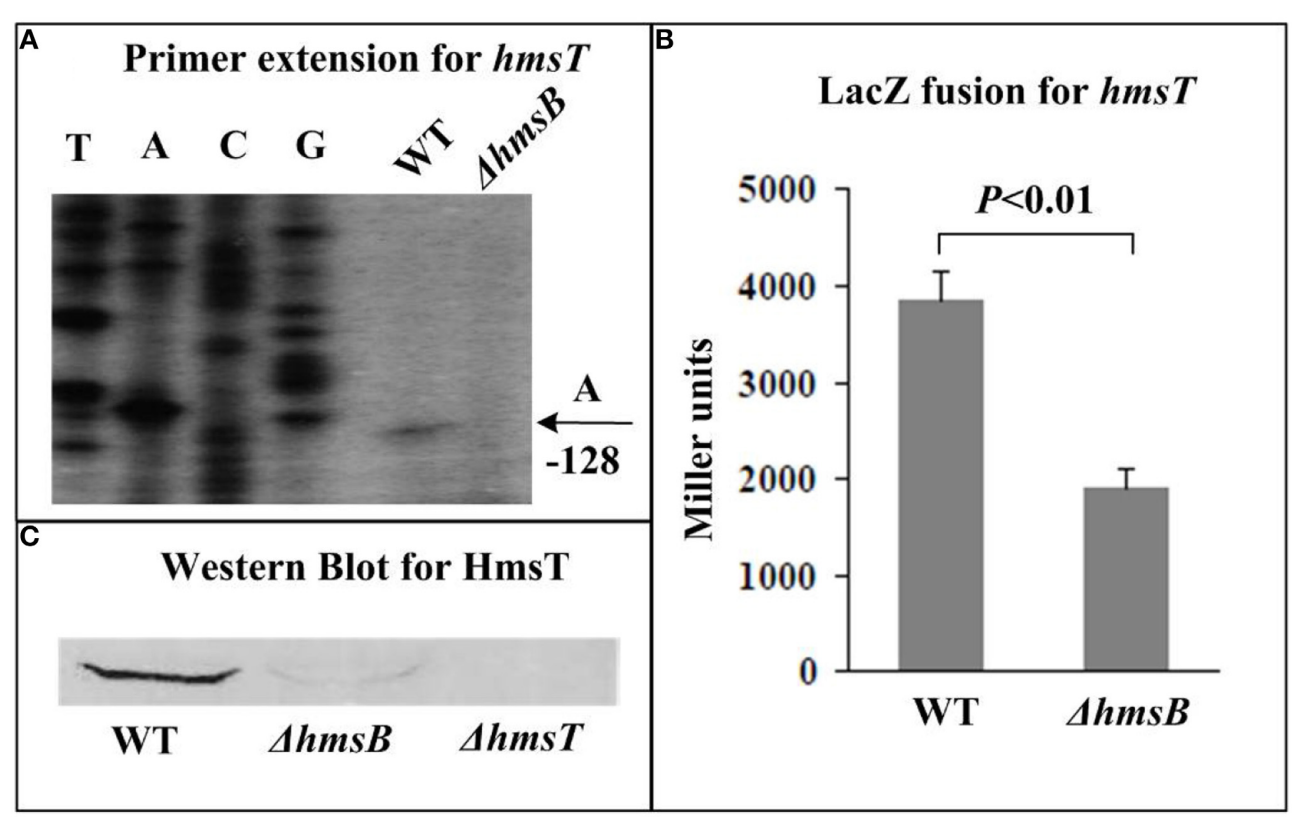

FIGURE 5 | HmsB-dependent expression of $\boldsymbol{h m s}$ T. Primer extension (A) and LacZ fusion (B) for $h m s T$, and Western Blot (C) for HmsT were done as described in Figure $\mathbf{3}$.

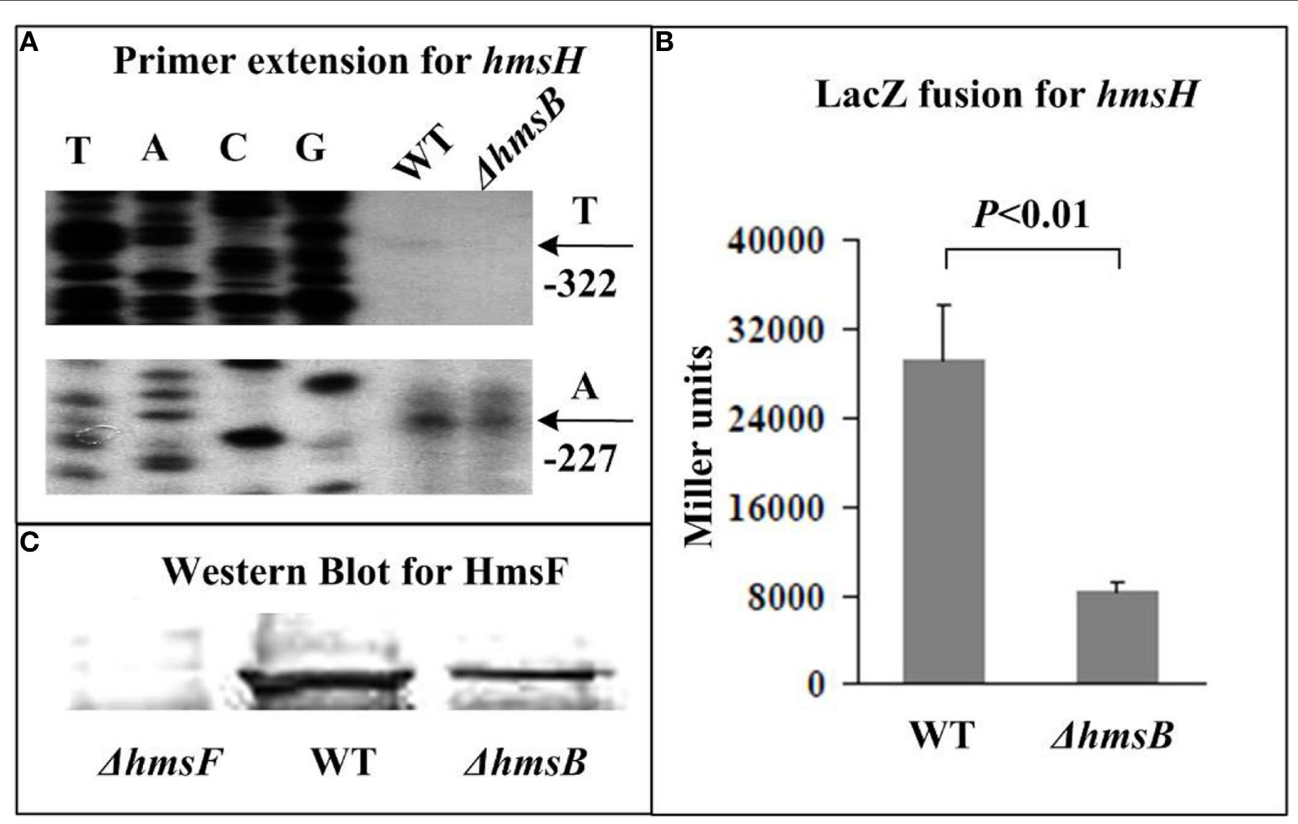

FIGURE 6 | HmsB-dependent expression of hmsHFRS. Primer

extension (A) and LacZ fusion (B) for hmsH, and Western Blot (C) for $\mathrm{HmsF}$ were done as described in Figure 3. Noted that the first gene

block ribosome binding and thus to inhibit protein biosynthesis (Han et al., 2013). Less commonly in cases studied to date, sRNAs can activate translation by freeing RBSs that would otherwise be occluded by inhibitory secondary structures (Han et al., 2013). Whether HmsB binds to RBS-around regions of these hms genes needs to be elucidated. of hmsHFRS was subjected to gene expression assays $(\mathbf{A}, \mathbf{B})$, while polysaccharide deacetylase HmsF was chosen for protein biosynthesis analysis (C).

The positive regulatory action of $\mathrm{HmsB}$ on the promoter activity of $h m s B, h m s C D E, h m s T$, and $h m s H F R S$, as characterized in this work, are highly unusual; it is speculated that HmsB modulates the translation of one or more transcriptional activators or repressors of the above hms genes. It should be noted that multiple transcriptional regulators of $Y$. pestis biofilm formation have 


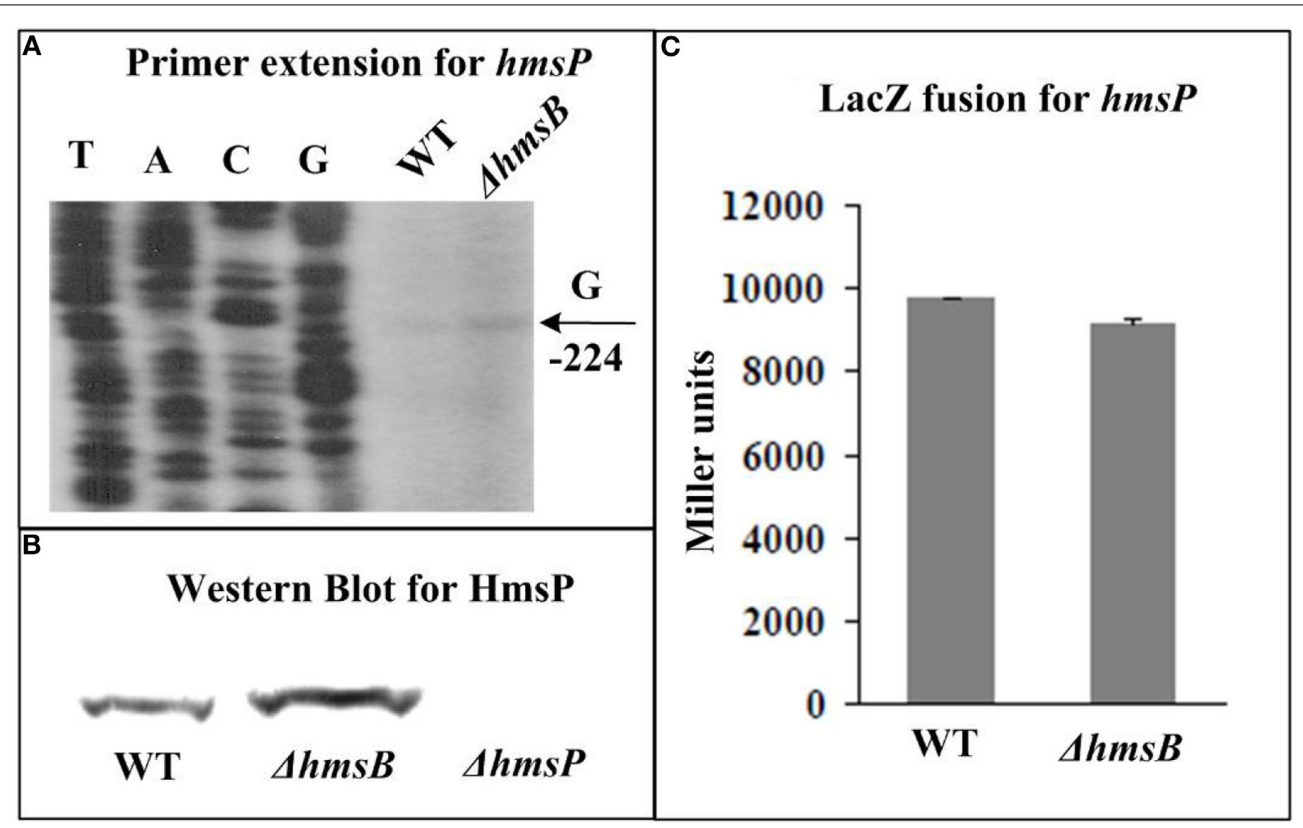

FIGURE 7 | HmsB-dependent expression of $\boldsymbol{h} \boldsymbol{m s} \boldsymbol{P}$. Primer extension (A) and LacZ fusion (C) for hmsH, and Western Blot (B) for HmsP were done as described in Figure 3. Noted that each of $h m s B$ (Figure 3), $h m s C D E$
(Figure 4), hmsT (Figure 5), and $h m s P$ (this figure) had a single transcription start site, while hmsHFRS (Figure 6) had two different ones (see our companion submission for details). been identified (Sun et al., 2012; Rebeil et al., 2013; Tam et al., 2014).

The $h f q$ deletion led to dramatic degeneration of HmsB in $Y$. pestis (unpublished data). Most of sRNAs characterized to date need binding of Hfq as a RNA chaperone, stabilizing formation of imperfect sRNA-target RNA duplexes (Han et al., 2013). It has been characterized that Hfq is essential for the biofilm formation and flea blockage of $Y$. pestis strain KIM6+ during colonization of flea gut (Rempe et al., 2012). Positive control of biofilm formation by Hfq is also observed in Y. pestis strain 201 used in this study, and further gene regulation assays show that Hfq enhancs the expression of $h m s C D E, h m s T$, and $h m s H F R S$ but inhibits that of $h m s P$ in this strain (unpublished data). By contrast, a separate study reports that $\mathrm{Hfq}$ is a repressor of biofilm formation through inhibiting expression of hmsCDE, hmsT, and hmsHFRS but stimulating that of $h m s P$ in a pCD1-derivate of $Y$. pestis CO92 (Bellows et al., 2012); interestingly, similar results can be observed in $Y$. pestis strain 201 cured of pCD1 (unpublished data).

The sRNA Ysr141 targets an untranslated region upstream of yopJ to posttranscriptionally activate synthesis of YopJ, an effector protein of the Yop-Ysc type III secretion system (Schiano et al., 2014); this is up to now the only report of sRNA-target gene association in $Y$. pestis. Although HmsB-dependent expression hms genes have been dissected in the present work, direct HmsB targets (probably including not only hms genes but their upstream transcriptional regulators) as well as detailed mechanisms of action of $\mathrm{HmsB}$ in aid of $\mathrm{Hfq}$ on its target genes needs to be dissected to understand how HmsB contributes to biofilm gene regulation.

\section{ACKNOWLEDGMENTS}

This work was supported by National Natural Science Foundation of China (81201246), Beijing Nova Program (Z121102002512049), and National Basic Research Program of China (Program 973, 2013CB910800). The English writing of this manuscript was polished by EnPapers.

\section{SUPPLEMENTARY MATERIAL}

The Supplementary Material for this article can be found online at: http://www.frontiersin.org/journal/10.3389/fmicb. 2014.00685/abstract

\section{REFERENCES}

Beauregard, A., Smith, E. A., Petrone, B. L., Singh, N., Karch, C., McDonough, K. A., et al. (2013). Identification and characterization of small RNAs in Yersinia pestis. RNA Biol. 10, 397-405. doi: 10.4161/rna.23590

Bellows, L. E., Koestler, B. J., Karaba, S. M., Waters, C. M., and Lathem, W. W. (2012). Hfq-dependent, co-ordinate control of cyclic diguanylate synthesis and catabolism in the plague pathogen Yersinia pestis. Mol. Microbiol. 86, 661-674. doi: $10.1111 / \mathrm{mmi} .12011$

Bobrov, A. G., Kirillina, O., Forman, S., Mack, D., and Perry, R. D. (2008). Insights into Yersinia pestis biofilm development: topology and co-interaction of $\mathrm{Hms}$ inner membrane proteins involved in exopolysaccharide production. Environ. Microbiol. 10, 1419-1432. doi: 10.1111/j.1462-2920.2007.01554.x

Bobrov, A. G., Kirillina, O., and Perry, R. D. (2005). The phosphodiesterase activity of the HmsP EAL domain is required for negative regulation of biofilm formation in Yersinia pestis. FEMS Microbiol. Lett. 247, 123-130. doi: 10.1016/j.femsle.2005.04.036

Bobrov, A. G., Kirillina, O., Ryjenkov, D. A., Waters, C. M., Price, P. A., Fetherston, J. D., et al. (2011). Systematic analysis of cyclic di-GMP signalling enzymes and their role in biofilm formation and virulence in Yersinia pestis. Mol. Microbiol. 79, 533-551. doi: 10.1111/j.1365-2958.2010.07470.x

Darby, C. (2008). Uniquely insidious: Yersinia pestis biofilms. Trends Microbiol. 16, 158-164. doi: 10.1016/j.tim.2008.01.005 
Darby, C., Hsu, J. W., Ghori, N., and Falkow, S. (2002). Caenorhabditis elegans: plague bacteria biofilm blocks food intake. Nature 417, 243-244. doi: $10.1038 / 417243 a$

Datsenko, K. A., and Wanner, B. L. (2000). One-step inactivation of chromosomal genes in Escherichia coli K-12 using PCR products. Proc. Natl. Acad. Sci. U.S.A. 97, 6640-6645. doi: 10.1073/pnas.120163297

Deng, Z., Liu, Z., Bi, Y., Wang, X., Zhou, D., Yang, R., et al. (2014). Rapid degradation of Hfq-free RyhB in Yersinia pestis by PNPase independent of putative ribonucleolytic complexes. Biomed. Res. Int. 2014:798918. doi: $10.1155 / 2014 / 798918$

Erickson, D. L., Jarrett, C. O., Wren, B. W., and Hinnebusch, B. J. (2006). Serotype differences and lack of biofilm formation characterize Yersinia pseudotuberculosis infection of the Xenopsylla cheopis flea vector of Yersinia pestis. J. Bacteriol. 188, 1113-1119. doi: 10.1128/JB.188.3.1113-1119.2006

Fang, N., Gao, H., Wang, L., Qu, S., Zhang, Y. Q., Yang, R. F., et al. (2013). Optimized methods for biofilm analysis in Yersinia pestis. Biomed Environ. Sci. 26, 408-411. doi: 10.3967/0895-3988.2013.05.012

Han, Y., Liu, L., Fang, N., Yang, R., and Zhou, D. (2013). Regulation of pathogenicity by noncoding RNAs in bacteria. Future Microbiol. 8, 579-591. doi: $10.2217 / \mathrm{fmb} .13 .20$

Hinnebusch, B. J., and Erickson, D. L. (2008). Yersinia pestis biofilm in the flea vector and its role in the transmission of plague. Curr. Top. Microbiol. Immunol. 322, 229-248. doi: 10.1007/978-3-540-75418-3_11

Kirillina, O., Fetherston, J. D., Bobrov, A. G., Abney, J., and Perry, R. D. (2004). HmsP, a putative phosphodiesterase, and HmsT, a putative diguanylate cyclase, control Hms-dependent biofilm formation in Yersinia pestis. Mol. Microbiol. 54, 75-88. doi: 10.1111/j.1365-2958.2004.04253.x

Koo, J. T., Alleyne, T. M., Schiano, C. A., Jafari, N., and Lathem, W. W. (2011). Global discovery of small RNAs in Yersinia pseudotuberculosis identifies Yersinia-specific small, noncoding RNAs required for virulence. Proc. Natl. Acad. Sci. U.S.A. 108, E709-E717. doi: 10.1073/pnas. 1101655108

Lodge, J., Fear, J., Busby, S., Gunasekaran, P., and Kamini, N. R. (1992). Broad host range plasmids carrying the Escherichia coli lactose and galactose operons. FEMS Microbiol. Lett. 74, 271-276. doi: 10.1111/j.1574-6968.1992. tb05378.x

McArthur, S. D., Pulvermacher, S. C., and Stauffer, G. V. (2006). The Yersinia pestis gcvB gene encodes two small regulatory RNA molecules. BMC Microbiol. 6:52. doi: 10.1186/1471-2180-6-52

Qu, Y., Bi, L., Ji, X., Deng, Z., Zhang, H., Yan, Y., et al. (2012). Identification by cDNA cloning of abundant sRNAs in a human-avirulent Yersinia pestis strain grown under five different growth conditions. Future Microbiol. 7, 535-547. doi: $10.2217 /$ fmb. 12.13

Rebeil, R., Jarrett, C. O., Driver, J. D., Ernst, R. K., Oyston, P. C., and Hinnebusch, B. J. (2013). Induction of the Yersinia pestis PhoP-PhoQ regulatory system in the flea and its role in producing a transmissible infection. J. Bacteriol. 195, 1920-1930. doi: 10.1128/JB.02000-12

Rempe, K. A., Hinz, A. K., and Vadyvaloo, V. (2012). Hfq regulates biofilm gut blockage that facilitates flea-borne transmission of Yersinia pestis. J. Bacteriol. 194, 2036-2040. doi: 10.1128/JB.06568-11

Schiano, C. A., Koo, J. T., Schipma, M. J., Caulfield, A. J., Jafari, N., and Lathem, W. W. (2014). Genome-wide analysis of small RNAs expressed by Yersinia pestis identifies a regulator of the Yop-Ysc type III secretion system. J. Bacteriol. 196, 1659-1670. doi: 10.1128/JB.01456-13

Straley, S. C., and Bowmer, W. S. (1986). Virulence genes regulated at the transcriptional level by $\mathrm{Ca} 2+$ in Yersinia pestis include structural genes for outer membrane proteins. Infect. Immun. 51, 445-454.

Sun, F., Gao, H., Zhang, Y., Wang, L., Fang, N., Tan, Y., et al. (2012). Fur is a repressor of biofilm formation in Yersinia pestis. PLoS ONE 7:e52392. doi: 10.1371/journal.pone.0052392

Sun, Y. C., Koumoutsi, A., Jarrett, C., Lawrence, K., Gherardini, F. C., Darby, C., et al. (2011). Differential control of Yersinia pestis biofilm formation In Vitro and in the flea vector by two c-di-GMP diguanylate cyclases. PLoS ONE 6:e19267. doi: 10.1371/journal.pone.0019267

Tam, C., Demke, O., Hermanas, T., Mitchell, A., Hendrickx, A. P., and Schneewind, O. (2014). YfbA, a Yersinia pestis regulator required for colonization and biofilm formation in the gut of cat fleas. J. Bacteriol. 196, 1165-1173. doi: 10.1128/JB.01187-13

Yan, Y., Su, S., Meng, X., Ji, X., Qu, Y., Liu, Z., et al. (2013). Determination of sRNA expressions by RNA-seq in Yersinia pestis grown in vitro and during infection. PLoS ONE 8:e74495. doi: 10.1371/journal.pone.0074495

Zhang, Y., Wang, L., Fang, N., Qu, S., Tan, Y., Guo, Z., et al. (2013a). Reciprocal regulation of $\mathrm{pH} 6$ antigen gene loci by $\mathrm{PhoP}$ and RovA in Yersinia pestis biovar Microtus. Future Microbiol. 8, 271-280. doi: 10.2217/fmb.12.146

Zhang, Y., Wang, L., Han, Y., Yan, Y., Tan, Y., Zhou, L., et al. (2013b). Autoregulation of PhoP/PhoQ and positive regulation of the cyclic AMP receptor proteincyclic AMP complex by PhoP in Yersinia pestis. J. Bacteriol. 195, 1022-1030. doi: 10.1128/JB.01530-12

Zhou, D., Tong, Z., Song, Y., Han, Y., Pei, D., Pang, X., et al. (2004). Genetics of metabolic variations between Yersinia pestis biovars and the proposal of a new biovar, microtus. J. Bacteriol. 186, 5147-5152. doi: 10.1128/JB.186.15.51475152.2004

Zhou, D., and Yang, R. (2011). Formation and regulation of Yersinia biofilms. Protein Cell 2, 173-179. doi: 10.1007/s13238-011-1024-3

Conflict of Interest Statement: The Associate Editor, Yicheng Sun, declares that, despite having collaborated with author, Dongsheng Zhou, on the same Research Topic, the review process was handled objectively. The authors declare that the research was conducted in the absence of any commercial or financial relationships that could be construed as a potential conflict of interest.

Received: 24 October 2014; accepted: 21 November 2014; published online: 12 December 2014.

Citation: Fang N, Qu S, Yang H, Fang H, Liu L, Zhang Y, Wang L, Han Y, Zhou D and Yang R (2014) HmsB enhances biofilm formation in Yersinia pestis. Front. Microbiol. 5:685. doi: 10.3389/fmicb.2014.00685

This article was submitted to Food Microbiology, a section of the journal Frontiers in Microbiology.

Copyright (c) 2014 Fang, Qu, Yang, Fang, Liu, Zhang, Wang, Han, Zhou and Yang. This is an open-access article distributed under the terms of the Creative Commons Attribution License (CC BY). The use, distribution or reproduction in other forums is permitted, provided the original author(s) or licensor are credited and that the original publication in this journal is cited, in accordance with accepted academic practice. No use, distribution or reproduction is permitted which does not comply with these terms. 